\title{
Thorax
}

Editorials

\section{High dose rate endobronchial brachytherapy for the treatment of lung cancer: current status and indications}

\author{
..."Diseases desperate grown, \\ By desperate appliance are reliev'd, \\ Or not at all - "
}

The last decade has seen the development of a number of "local" - that is, endobronchial - treatment modalities for lung cancer, with the recognition that the endobronchial component is not only responsible for much of the symptomatology but is also accessible to direct examination and intervention. Useful techniques range from those of a principally "physical" nature such as endoscopic surgery and diathermy, laser photoresection, cryotherapy and stent placement, to treatments which may be regarded as more "biological" in nature, including photodynamic therapy and brachytherapy.

Endobronchial brachytherapy is not new; as long ago as 1922 Yankauer treated two patients by bronchoscopic placement of encapsulated radium sources. More recently the development of flexible bronchoscopy, miniature high dose rate radioisotope technology, and remote afterloading systems has enabled extremely active intrabronchial therapy to be administered in a safer, more precise, and more controlled fashion than has hitherto been possible.

The technique ${ }^{2}$ is now well known; a miniature radioactive source, welded to its drive cable, is delivered by remote control from a "safe" to a series of preplanned positions inside a catheter, placed for the purpose either within or alongside an endobronchial tumour. The catheter is placed using a standard flexible fibreoptic bronchoscope. Some series have reported laser disobliteration of the bronchial lumen before catheter placement but in our experience this has rarely been necessary. The size of the effective treatment volume is determined by the number of sequential positions of the source and by the dwell-time at each position. Typically, a sausage-shaped volume some $10 \mathrm{~cm}$ in length and $2 \mathrm{~cm}$ in diameter may be irradiated using a single catheter. If necessary two or more catheters can be used to create a more complex shape with better conformation to tumour volume, in which case computerised dose optimisation is helpful. The treatment is intense but localised, sparing the oesophagus, spinal cord, and surrounding lung from the possible effects of external beam radiotherapy. Treatment times are short with the high dose rate system, often a mere 10 minutes or so per fraction; the technique is thus suitable for outpatient or day case therapy.

The treatment is effective in achieving local tumour control. Assessment by repeat endoscopic examination indicates a delayed but progressive and often considerable reduction in tumour volume, complete endoscopic remission being quite common. Convincing relief of cough, breathlessness and, particularly, haemoptysis may be obtained with concomitant improvement in performance status, ${ }^{2-4}$ but published data on more detailed quality of life assessments are sparse. Re-expansion of collapsed lung tissue occurs in about $50 \%$ of patients, a figure comparable with external beam radiotherapy. Improvement in pulmonary function, ventilation and perfusion, and exercise tolerance has been reported. ${ }^{5}$

What are the current indications for brachytherapy? The localised nature of the treatment renders it suitable for the palliation of symptoms and the relief of obstructive collapse where these are caused by intrabronchial tumour. Comparative data are surprisingly rare, but there is evidence from our own series that in this group its overall efficacy is comparable with that of fractionated external beam regimens which are more demanding of patient and therapist time. No benefit can be anticipated from brachytherapy alone in patients with bulk disease causing extrinsic bronchial compression or more distant effects such as somatic pain and dysphagia. For primary palliation the duration of response is impressive, more than two thirds of patients requiring no additional radiotherapy during their lifetime. ${ }^{6}$ The other third will require retreatment, usually with conventional radiotherapy.

Primary palliation is therefore its clearest role at the present time. It does, however, offer additional flexibility for retreatment of recurrent or residual disease, ${ }^{46-8}$ for contralateral or multiple primary tumours, combined therapy, or in patients with poor pulmonary function in whom radiation fibrosis might be expected to be poorly tolerated.

A few patients have prolonged disease-free survival even though the treatment was initially intended to be palliative. About $1 \%$ of the Manchester series falls into this category, and a number of authors have commented on the prolonged survival and curative potential ${ }^{910}$ where a tumour is small and capable of being totally included in the treatment volume. Accurate assessment of the volume and extent of small tumours is difficult however, even with the assistance of high resolution computed tomographic scanning. No comparative radical treatment data are available, most reports being small pilot studies or even individual case reports. The current position is that, although brachytherapy has undoubted curative potential, it is not appropriate to regard it as a primary treatment if other forms of radical treatment are practicable. There remains a small group which may be legitimately studied - those patients with technically operable or radically treatable disease who are unfit for surgery or external beam therapy for a number of reasons, the commonest being poor respiratory function. 
A treatment of such local intensity is inevitably capable of causing a number of local side effects. Placement of the applicator may cause transient pleuritic pain and, occasionally, pneumothorax. Many patients experience a transient increase in cough. Radiation bronchitis causes a more persistent cough and may lead to the formation of a membranous exudate with subsequent concentric stenosis of the airway. A more functionally significant stenosis of the larger airways may occasionally occur requiring debridement or stent placement. ${ }^{11}$ These reactions are more frequent in patients treated with concomitant radiotherapy, in whom it is common to see dense collagenous "tracks" where the catheter has been in direct contact with normal mucosa rather than being "centred" by intrabronchial tumour. New applicator designs which incorporate spacers to keep the applicator in the centre of the bronchial lumen are currently being evaluated. ${ }^{12}$

A major concern is the possibility of fistula formation and haemorrhage, particularly the incidence of massive haemoptysis as a terminal event following treatment. The reported incidence varies from zero to $50 \%,{ }^{13}$ several series reporting around one third. ${ }^{814}$ The higher rates are found where patients have received prior or concurrent high dose external beam irradiation or laser ${ }^{15}$ therapy. The incidence of massive fatal haemoptysis in our own series was $7.9 \%$ at a median interval of 214 days after treatment. Multivariate analysis of associated factors suggested a correlation with a higher initial dosage, prior laser therapy, or a second intrabronchial treatment, concurrent external beam radiotherapy approaching statistical significance. ${ }^{16}$

These figures are clearly a cause for anxiety but are mitigated by a number of factors. In the first place, bronchial carcinoma can itself cause fatal haemoptysis with a probable incidence of about 3\%. ${ }^{17}$ Secondly, necroscopic studies show that nearly all patients who have fatal bleeding following brachytherapy do have recurrent disease. Thirdly, there are indications that this fatal complication tends to occur in patients who have a longer overall survival.

It is unfortunate that the manner in which the technique has been investigated and developed worldwide has left many questions unanswered. Although the dose used is now more or less universally specified at $1 \mathrm{~cm}$ radius from the centre of the applicator, the total treatment length and volume are frequently unreported. A wide variety of total doses, fractionation schedules, and treatment combinations has been used. Many studies which appear to record the effects of brachytherapy are in fact reporting those of combined treatment or of salvage treatment in previously irradiated patients. There are few data which compare brachytherapy with external beam radiotherapy, neither has it been shown that the addition of brachytherapy to external beam treatment improves the symptomatic response, local control or survival, though there is some evidence that complication rates may be higher. We have used a single fraction of $15 \mathrm{~Gy}$, a safe, effective, and convenient regimen using brachytherapy alone; most studies have used two or more fractions in combination with external irradiation. There is some evidence that the total dose and the dose per fraction may influence morbidity in these patients. ${ }^{318}$ Our own morbidity data suggest that a single dose of $20 \mathrm{~Gy}$ at a radius of $1 \mathrm{~cm}$, or its biological equivalent in fractionated schedules, may be close to tissue tolerance. Clearly, for patients treated palliatively we should be seeking the lowest biological dose capable of achieving a worthwhile response. Randomised studies are required to clarify some of these issues.

On pages $354-8$ of this issue of Thorax Hernandez et al report the effect of brachytherapy on local control in patients who have already received external beam irradiation. ${ }^{19}$ The study is a prospective one, with patients assessed in considerable detail including repeat bronchoscopic examination and performance status in addition to symptom scoring and chest radiographs. There is also an attempt to relate the overall response to the degree of bronchoscopic remission obtained. The authors have shown that a worthwhile symptomatic response can be obtained in previously treated patients, particularly with respect to haemoptysis. The relief of other symptoms was disappointing but was better if there was evidence of endoscopic remission. Performance status improved in $24 \%$ of patients irrespective of endoscopic response. As with previous studies, ${ }^{4-8}$ the results suggest that brachytherapy can be effective as secondary palliation, but the study design offers no opportunity for comparison with either best supportive care or with other treatment modalities. The follow up period of four weeks (eight for repeat endoscopy) was too short to allow any valid assessment of serious treatment-related morbidity such as massive fatal haemoptysis or radiation bronchitis.

Finally, what is the place of brachytherapy in relation to other intrabronchial techniques? Laser photoresection and endobronchial surgery (with or without diathermy) can achieve more rapid relief of large airway obstruction and can be used in a situation where radiation therapy may be contraindicated. One example is the presence of critical tracheal stenosis where post-radiation oedema may aggravate stridor before alleviating it. The response to brachytherapy is considerably slower than to laser resection but is progressive and durable, retreatment being required less frequently. Endoscopic surgical resection, laser photoresection or stent placement may, however, be used to relieve a critical stenosis rapidly and to allow a more definitive radiotherapy treatment to be completed. Direct comparative studies of YAG laser and brachytherapy are probably now inappropriate as most therapists with access to both regard them as complementary techniques. Physical disobliteration methods and stent placement may be used to salvage brachytherapy relapses but many patients in this situation will by then have advanced disease and will obtain only limited benefit.

Photodynamic therapy remains a biological alternative to endobronchial radiation, particularly as a potentially curative technique for small tumours. Few centres are equipped to conduct a comparative study of this nature and photodynamic therapy has been slow to gain widespread acceptance. The development of more tumour-specific, and therefore less toxic, systemic sensitisers may alter this.

In summary, intrabronchial radiation is highly effective for the primary palliation of inoperable lung cancer, either alone or in combination with external beam radiotherapy. The technique is also effective for the secondary palliation of relapsed disease and considerably increases the overall scope for the irradiation of lung tumours. Tumour response to treatment is delayed but ultimately more complete and more durable than that obtained with physical disobliteration techniques, though some patients will require these in addition. The role of brachytherapy alone as a radical treatment is not yet validated but it is likely to have curative potential for small tumours. Optimal total dose, fractionation, and relationship to external beam therapy have yet to be established, but a number of randomised studies are now in progress.

Correspondence to: Dr P Barber.

North West Lung Centre,

Wythenshawe Hospital

Manchester M23 9LT, UK

Christie Hospital,

Manchester M20 4BX, UK
PHIL BARBER

RON STOUT 
1 Hamlet IV,iii,9.

Burt P, O'Driscoll B, Notley H, Barber P, Stout R. Intraluminal irradiation for the palliation of lung cancer with high dose rate Micro-Selectron Thorax 1990;45:765-8.

3 Dattatreyudu Nori, Allison R, Kaplan Barbi, Samala Engracio, Osian A, Karbowitz S. High dose-rate intraluminal irradiation in bronchogenic carcinoma. Chest 1993;104:1006-11.

4 Speiser BL, Spratling L. Remote afterloading brachytherapy for the local control of endobronchial carcinoma. Int f Radiat Oncol Biol Phys 1993; 25:579-87.

5 Goldman JM, Bulman AS, Rathmell AJ, Carey BM, Muers MF, Joslin CAF. Physiological effect of endobronchial radiotherapy in patients with major airway occlusion by carcinoma. Thorax 1993;48:110-4.

6 Gollins SW, Burt PA, Barber PV, Stout R. High dose rate intraluminal radiotherapy for carcinoma of the bronchus: outcome of treatment of 406 radiotherapy for carcinoma of the bronchus:
patients. Radiother Oncol 1994;33:31-40.

patients. Radiother Oncol 1994;33:31-40.
7 Pisch J, Villamena PC, Harvey JC, Rosenblatt E, Mishra S, Beattie EJ. High dose-rate endobronchial irradiation in malignant airway obstruction. High dose-rate endobro

8 Bedwineck J, Petty A, Bruton C, Sofield J, Lee L. The use of high dose rate endobronchial brachytherapy to palliate symptomatic endobronchial recurrence of previously irradiated bronchogenic carcinoma. Int $\mathcal{f}$ Radiat Oncol Biol Phys 1992;22:23-30.

9 Sutedja G, Baris G, Zandwijk N Van, Postmus PE. High dose rate brachytherapy has a curative potential in patients with intraluminal squamous cell lung cancer. Respiration 1993;61:167-8.

10 Tredaniel J, Hennequin C, Zalcman G, Walter S, Homasson JP, Maylin C, et al. Prolonged survival after high-dose rate endobronchial radiation for malignant airway obstruction. Chest 1994;105:767-72.
11 Speiser BL, Spratling L. Radiation bronchitis and stenosis secondary to high dose rate endobronchial irradiation. Int $\mathcal{f}$ Radiat Oncol Biol Phys 1993;25:589-97.

12 Nomoto Y, Shoji K, Sasoaka M, Nakagawa T, Toyota S. Proceedings of Eighth International Brachytherapy Conference, Nice, November 1995, Old Delft, The Netherlands: Nucletron, 1995:288(abstract 123).

13 Khanavkar B, Stern P, Alberti W, Nakhosteen JA. Complications associated with brachytherapy alone or with laser in lung cancer. Chest 1991;99: 1062-5.

14 Seagren S, Harrell HJ, Horn RA. High dose rate intraluminal irradiation in recurrent endobronchial carcinoma. Chest 1985;88:810-4

15 Mehta M, Shahabi S, Jarjour N, Steinmetz M, Kubsad S. Effect of endobronchial radiation therapy on malignant bronchial obstruction. Chest 1990;97:662-5.

16 Burt PA, Barber PV, Collins SW, Stout R. Morbidity associated with HDR intraluminal radiotherapy for carcinoma of the bronchus: Christie Hospital experience. Proceedings of Eight International Brachytherapy Conference, Nice, November 1995, Old Delft, The Netherlands: Nucletron, 1995: 138(abstract 55).

17 Miller R, McGregor D. Haemorrhage from carcinoma of the lung. Cancer 1980;46:200-5.

18 Chang LFL, Horvath J, Peyton W, Ling SS. High dose rate afterloading intraluminal brachytherapy in malignant airway obstruction of lung cancer. Int $\mathcal{F}$ Radiat Oncol Biol Phys 1994;28:589-96.

19 Hernandez P, Gursahaney A, Roman T, Schwartzman K, Donath D, Cosio MG, et al. High dose rate brachytherapy for the local control of endobronchial carcinoma following external irradiation. Thorax 1996;51: 354-8. 